\title{
Remanent magnetization in $\mathrm{CoO}$ antiferromagnetic nanoparticles
}

\author{
N. J. O. Silva, * A. Millán, and F. Palacio \\ Instituto de Ciencia de Materiales de Aragón, CSIC-Universidad de Zaragoza, 50009 Zaragoza, Spain \\ and Departamento de Física de la Materia Condensada, Facultad de Ciencias, Universidad de Zaragoza, 50009 Zaragoza, Spain
}

M. Martins and T. Trindade
Departamento de Química and CICECO, Universidade de Aveiro, 3810-193 Aveiro, Portugal

I. Puente-Orench and J. Campo

Instituto de Ciencia de Materiales de Aragón, CSIC-Universidad de Zaragoza, 50009 Zaragoza, Spain

and Institut Laue-Langevin, BP 156, F-38042 Grenoble Cedex 9, France

(Received 18 May 2010; revised manuscript received 23 July 2010; published 20 September 2010)

\begin{abstract}
We report a study on the remanent magnetization $M_{r}$ induced by field cooling across the ordering temperature $T_{N}$ in antiferromagnetic $\mathrm{CoO}$ nanoparticles with different sizes. The nanoparticles are composed by a structurally and magnetically ordered core and a structurally ordered and magnetically disordered shell with a thickness of about $2 \mathrm{~nm}$. The ordered core has cell parameters, moments direction, and modulus similar to those of bulk CoO. $M_{r}$ is shown to be proportional to the cooling field $H_{\text {cool }}$. The low-temperature saturation values of $M_{r}\left[M_{r}(0)\right]$ in the $\mathrm{CoO}$ nanoparticles are about two orders of magnitude higher than those found for bulk CoO. $M_{r} / M_{r}(0)$ of $\mathrm{CoO}$ nanoparticles scales with temperature in a single curve, independently on the magnitude of $H_{\text {cool }}$ and on nanoparticles size, except for temperatures near to $T_{N}$ since $T_{N}$ is size dependent.
\end{abstract}

DOI: 10.1103/PhysRevB.82.094433

PACS number(s): 75.30.Cr, 75.50.Ee, 75.50.Tt

\section{INTRODUCTION}

It is known that bulk antiferromagnetic (AF) materials can exhibit remanent magnetization $M_{r}$. This is the case of $3 d$ random-field AF (Ref. 1) and of AF compounds where magnetic sites are diluted with nonmagnetic impurities, ${ }^{2}$ for instance. The origin of $M_{r}$ in the former is spin readjustments within the domain walls of immobile AF domains (at low temperatures) being $M_{r}$ also affected by domain growth at higher temperatures. ${ }^{1}$ In AF materials diluted with nonmagnetic impurities, the saturation of $M_{r}$ occurs at fields on the order of a few oersteds and the proposed mechanism is the pinning of domain walls due to the nonmagnetic impurities and lattice defects. ${ }^{2}$

Small nanoparticles (NPs) are expected to be monodomain and therefore the bulk mechanisms for the generation of $M_{r}$ associated to domain walls are not effective. Alternatively, $M_{r}$ can arise associated with uncompensated and canted moments due to surface and/or in-volume effects. In NPs, much attention has been paid to the interaction between $\mathrm{AF}$ and ferromagnetic components in the context of exchange bias (see, for instance the review in Ref. 3) but less to the existence of a $M_{r}$ associated to the AF component, ${ }^{4,5}$ although it was recognized that the $M_{r}$ of the AF component and the associated uncompensated moments have an important role in the exchange bias phenomena. ${ }^{4,6}$ In most of the cases, the largest fraction of $M_{r}$ is given by a ferromagnetic/ ferrimagnetic component, and the role of the AF component is to keep the moment in a fixed direction by increasing the coercivity and the blocking temperature of the NPs. ${ }^{7}$ In a recent example, the AF component was also found to increase the ordering temperature of the ferrimagnetic component. ${ }^{8}$

Considering surface vs in-volume effects, most studies are devoted to ferrimagnetic nanoparticles, where unlike in AF
NPs, surface is the origin of a reduction in the magnetic moment. These studies are not unanimous with some authors claiming the preferential surface canting 9,10 in disagreement with others where finite-size/in-volume effects are observed. ${ }^{11,12}$ Studies comparing surfactant-coated particles with uncoated ones show that the magnetic properties are greatly affected by the surfactant-particle interaction, suggesting the surface nature of this phenomena. ${ }^{13,14}$ Another attempt to characterize the origin of the uncompensated moment consists in comparing the particle size obtained from the electron microscopy or x-ray diffraction (XRD) techniques with the magnetic size derived from the field dependence of the magnetization, being the latter generally lower. ${ }^{15}$

In AF NPs, surface is often invoked as the origin of uncompensated moments. ${ }^{5,16-18}$ Evidence for the surface origin includes the linear decreasing of the saturation magnetization with temperature. ${ }^{16,19-21}$ The uncompensated moments include those arising from an odd number of AF planes, generation of vacancies and oxidation of surface atoms. ${ }^{5}$ In studies where surface effects are highlighted, the nanoparticles are modeled as having a magnetic ordered core surrounded by a magnetically disordered shell whose thickness is estimated as about $1 \mathrm{~nm} .{ }^{22-26}$ These studies were performed in ferrimagnetic materials including $\mathrm{MnFe}_{2} \mathrm{O}_{4},{ }^{22} \mathrm{CoFe}_{2} \mathrm{O}_{4},{ }^{23}$ and $\gamma-\mathrm{Fe}_{2} \mathrm{O}_{3},{ }^{24-26}$ and the techniques used have been magnetization, ${ }^{22,25,26}$ Mössbauer spectroscopy, ${ }^{25}$ and neutron diffraction. ${ }^{23}$ In particular, the study of polarized neutron diffraction in surfactant coated and uncoated $\mathrm{CoFe}_{2} \mathrm{O}_{4}$ nanoparticles provided a better insight on the radial distribution of the magnetization. ${ }^{23}$ The authors analyzed the XRD (400) reflection of this material to derive a crystalline particle size distribution and the same reflection in polarized neutron diffraction to extract the magnetically ordered size, smaller than the crystalline one, which corresponds to a magnetically dis- 
ordered shell of about 1.2 and $1.7 \mathrm{~nm}$ thick. ${ }^{23}$

Here we report on the behavior of $M_{r}$ induced by field cooling (fc) with different field intensities across $T_{N}$ in $\mathrm{CoO}$ AF NPs of different sizes. The observed behavior is compared with that exhibit by a sample with bulk properties. Powder neutron-diffraction (PND) technique has been used as a function of temperature to investigate the relation between magnetic and structural parameters of the NP and the origin of the moments responsible for $M_{r}$.

\section{EXPERIMENTAL}

The $\mathrm{CoO}$ nanoparticles were prepared by adaptation of the method of Sun et al. ${ }^{27}$ using cobalt acetate as the source of cobalt instead of cobalt formate. The size of the particles was adjusted by changing the cobalt to oleylamine ratio and the heating rate. In a typical synthesis, $\mathrm{Co}\left(\mathrm{CO}_{2} \mathrm{CH}_{3}\right)_{2}$ was dispersed in oleylamine (in concentration ratios ranging from $1 / 12$ to $1 / 48$ ) and the mixture was heated at $100{ }^{\circ} \mathrm{C}$ for 30 min under $\mathrm{N}_{2}$ atmosphere while repeatedly evacuated to remove oxygen and water. The solution was then heated up to $190{ }^{\circ} \mathrm{C}$ at different heating rates, from 10 to $20{ }^{\circ} \mathrm{C} / \mathrm{min}$, and kept for $3 \mathrm{~h}$ under vigorous magnetic stirring. After cooling the resulting solution to room temperature, the dark-brown suspension formed was precipitated by adding ethanol and repeatedly washed.

The sample with $\mathrm{CoO}$ nanoparticles with size $\langle\tau\rangle_{\mathrm{XRD}}$ $=24 \mathrm{~nm}$ was prepared by a different method; the decomposition of cobalt cupferronate $\left[\mathrm{Co}(\mathrm{cup})_{2}\right]$ in octadecene, accordingly to Ref. 28. Briefly, $0.25 \mathrm{~g}$ of $\mathrm{Co}(\mathrm{cup})_{2}$ were added to $25 \mathrm{~mL}$ of octadecene and the mixture was sealed in a Teflon-lined stainless-steel autoclave. The autoclave was kept inside a preheated air oven at $250{ }^{\circ} \mathrm{C}$ for $48 \mathrm{~h}$. After cooling to room temperature, the brownish-black solid was washed with toluene followed by methanol. The sample with $\mathrm{CoO}$ particles with larger size $\left(\langle\tau\rangle_{\mathrm{XRD}}=190 \mathrm{~nm}\right)$ is a comercial polycrystalline powdered sample obtained from SigmaAldrich.

Transmission electron microscopy (TEM) was performed using a Jeol-2000 FXII microscope, with point-to-point and line-to-line resolutions of $0.28 \mathrm{~nm}$ and $0.14 \mathrm{~nm}$, respectively, and equipped with a INCA 200 X-Sight (Oxford Instruments) energy dispersive x-ray spectrometer (EDS). Highresolution TEM (HRTEM) images were obtained in a FEI TECNAI G2 F30 instrument. Samples for TEM observations were prepared by dispersing the nanoparticles in hexane and evaporating suspension drops on carbon-coated copper grids.

XRD measurements of $\mathrm{CoO}$ samples were performed at room temperature with a Philips X'Pert-MPD diffractometer using monochromated $\mathrm{Cu} K \alpha$ radiation $(\lambda=1.541 \AA)$ in the $30^{\circ}-80^{\circ} 2 \theta$ range at $0.04^{\circ}$ resolution and 4000 acquisition points per step. The incident-beam optics included a Soller slit of $0.04 \mathrm{rad}$, a $10 \mathrm{~mm}$ fixed mask, a divergence fixed slit of $0.5^{\circ}$ and an antiscatter slit of $1^{\circ}$. The diffracted beam optics included a Soller slit of $0.04 \mathrm{rad}$ and antiscatter slit of $6.6 \mathrm{~mm}$. The analysis of the diffraction patterns was performed by Rietveld refinement using the FULLPROF package. ${ }^{29}$ The size effects were treated with the integral breadth method using the Voigt model for both the instru- mental and intrinsic diffraction peak shape considering a Thompson-Cox-Hastings pseudo-Voigt convoluted with axial divergence asymmetry function to describe the peak shape. The contribution of the instrument to the peaks broadening was determined by the refinement of the XRD pattern of a $\mathrm{LaB}_{6}$ standard sample (NIST Ref. 660a). The contribution of the finite size of the nanoparticles crystallites to the peaks broadening was taken into account by an isotropic model yielding an apparent size, proportional to the mean size of the structurally ordered region of the nanoparticles $\langle\tau\rangle_{\mathrm{XRD}}{ }^{29,30}$ In the following, samples will be identified by their $\langle\tau\rangle_{\mathrm{XRD}}$ value.

PND was performed in the powder diffractometers D20 and D1A of the Institute Laue Langevin (ILL). At D20, the experiments were carried out using a monochromatic beam of $1.87 \AA$. Neutron diffractograms were recorded as a function of the temperature and magnetic field by means of introducing the sample in a standard vanadium can inside an ILL cryomagnet. Diffraction patterns from 2 to $297 \mathrm{~K}$ were collected at zero field, after zero-field cooling (zfc) and after fc the samples from 297 to $200 \mathrm{~K}$ under a magnetic field of $1 \times 10^{4}$ Oe applied perpendicular to the neutron beam. In order to have neutron data with less instrumental contribution to the peaks broadening and better accuracy in the determination of the nuclear and magnetic mean sizes, a PND pattern at $5 \mathrm{~K}$ was collected at the instrument D1A with a monochromatic beam of $1.90 \AA$. The analysis of the PND patterns was performed as described for the XRD patterns. The contribution of the instrument to the peaks broadening was determined by the instrument resolution function built from the refinement of a $\mathrm{Na}_{2} \mathrm{Ca}_{3} \mathrm{Al}_{2} \mathrm{~F}_{14}$ standard sample while wavelengths were refined using a $\mathrm{Si}$ standard.

Magnetization was measured as a function of temperature from 2 to $300 \mathrm{~K}$ under a magnetic field of 100 Oe with a magnetic property measuring system (MPMS) model XL, Quantum Design Inc, equipped with a superconducting quantum interference device magnetometer. Two cooling procedures were used (i) zfc and (ii) field cooling across $T_{N}$, from 300 to $210 \mathrm{~K}$ and zfc from 210 to $2 \mathrm{~K}$. The field cooling in the restricted temperature range near $T_{N}$ of $\mathrm{CoO}$ was used to guarantee that only the moments associated to $\mathrm{CoO}$ are ordered in the presence of a field and not moments of a possible residual $\mathrm{Co}_{3} \mathrm{O}_{4}$ phase, which order at $\sim 40 \mathrm{~K}$. The remanent magnetization $M_{r}$ was calculated from the difference between the magnetization obtained in (ii) and (i).

\section{RESULTS AND DISCUSSION}

\section{A. Magnetic and nuclear structure}

Rietveld analysis to XRD patterns [Fig. 1(a)] confirms the existence of $\mathrm{CoO}$ nanoparticles with mean structural coherence size $\langle\tau\rangle_{\mathrm{XRD}}$ ranging from 18 to $68 \mathrm{~nm}$. Sample from Sigma-Aldrich has a $\langle\tau\rangle_{\mathrm{XRD}}=190 \mathrm{~nm}$. Any possible amount of $\mathrm{Co}_{3} \mathrm{O}_{4}$ present in the samples is below the detection limits.

TEM images of the sample with smaller nanoparticles $\left(\langle\tau\rangle_{\mathrm{XRD}}=18 \mathrm{~nm}\right)$ show that the $\mathrm{CoO}$ samples are composed of rhomboidal shaped nanoparticles with sizes on the order of 10 to $20 \mathrm{~nm}$ and some aggregates of these nanoparticles 


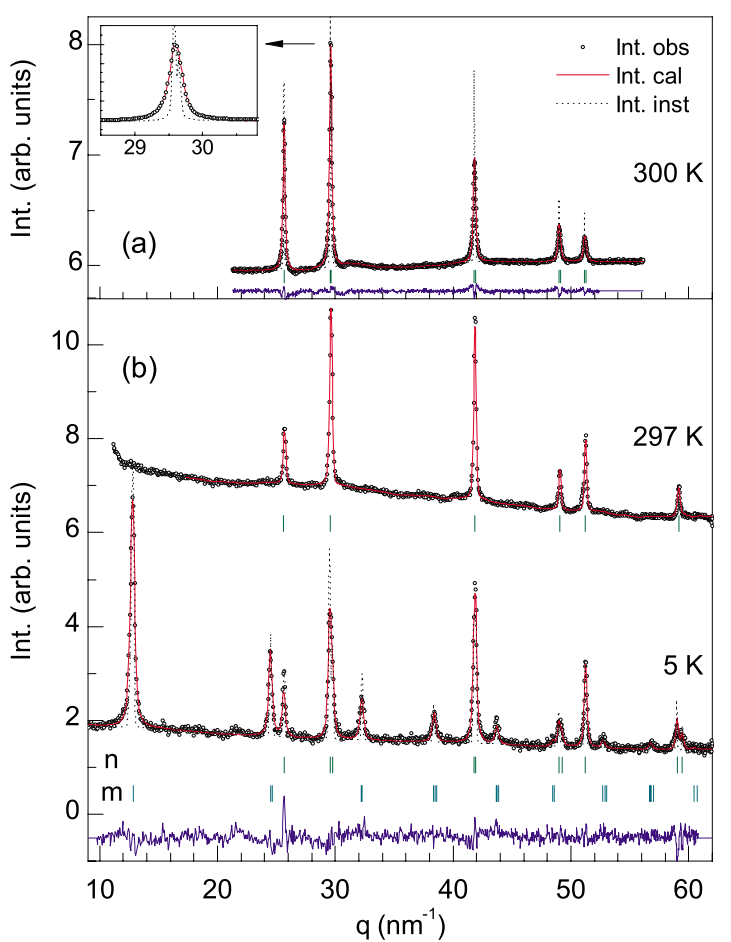

FIG. 1. (Color online) (a) XRD pattern of the $\mathrm{CoO}$ nanoparticles (sample with $\langle\tau\rangle_{\mathrm{XRD}}=33 \mathrm{~nm}$ ) at $300 \mathrm{~K}$. Vertical lines represent the position of the allowed Bragg peaks. Inset shows a zoom over the most intense peak. (b) PND pattern of the $\mathrm{CoO}$ nanoparticles (sample with $\langle\tau\rangle_{\mathrm{XRD}}=40 \mathrm{~nm}$ ) at $297 \mathrm{~K}$ and at $5 \mathrm{~K}$ (instrument D20 and D1A, respectively). Vertical lines represent the position of the allowed Bragg peaks of nuclear (n) and magnetic (m) origin. In both panels, the instrument contribution to the peaks shape is shown in dotted lines and Rietveld refinement considering the finite-size effect of the nanoparticles to the peaks shape is shown in continuous (red) lines.

[Figs. 2(a) and 2(b)]. Electron-diffraction patterns show rings and spots at the distances expected for $\mathrm{CoO}$ while EDS gives a molar proportion of $\mathrm{Co}$ and $\mathrm{O}$ of 1:1.15, consistent with a slightly oxidized $\mathrm{CoO}$. The sample with $\langle\tau\rangle_{\mathrm{XRD}}=40 \mathrm{~nm}$ consists of rhomboidal shaped aggregates with sizes of the order of $100 \mathrm{~nm}$ [Fig. 2(c)]. These aggregates are composed of smaller crystallites oriented in well-defined directions as deduced from the well-defined spots of electron diffraction [Fig. 2(d)].

HRTEM images of sample $\langle\tau\rangle_{\mathrm{XRD}}=18 \mathrm{~nm}$ show that the nanoparticles are single domain with planar boundaries and planes ordered up to the surface within the precision of the surface determination, estimated to be better than $0.4 \mathrm{~nm}$ [Fig. 3(a)]. The Fourier transform of the HRTEM images confirm that the nanoparticles are single domain. As expected, different zone axis are observed depending on the orientation of the nanoparticle, including the [2011] axis shown in Fig. 3(b).

The PND patterns performed in samples with $\langle\tau\rangle_{\mathrm{XRD}}$ $=40$ and $68 \mathrm{~nm}$ at room temperature confirm the information given by XRD, showing the peaks of the cubic face-centered $\mathrm{CoO}$ (space group $F m \overline{3} m$ ). ${ }^{31}$ As temperature decreases bellow $T_{N}$ (which is in the $250-290 \mathrm{~K}$ range and depends on the
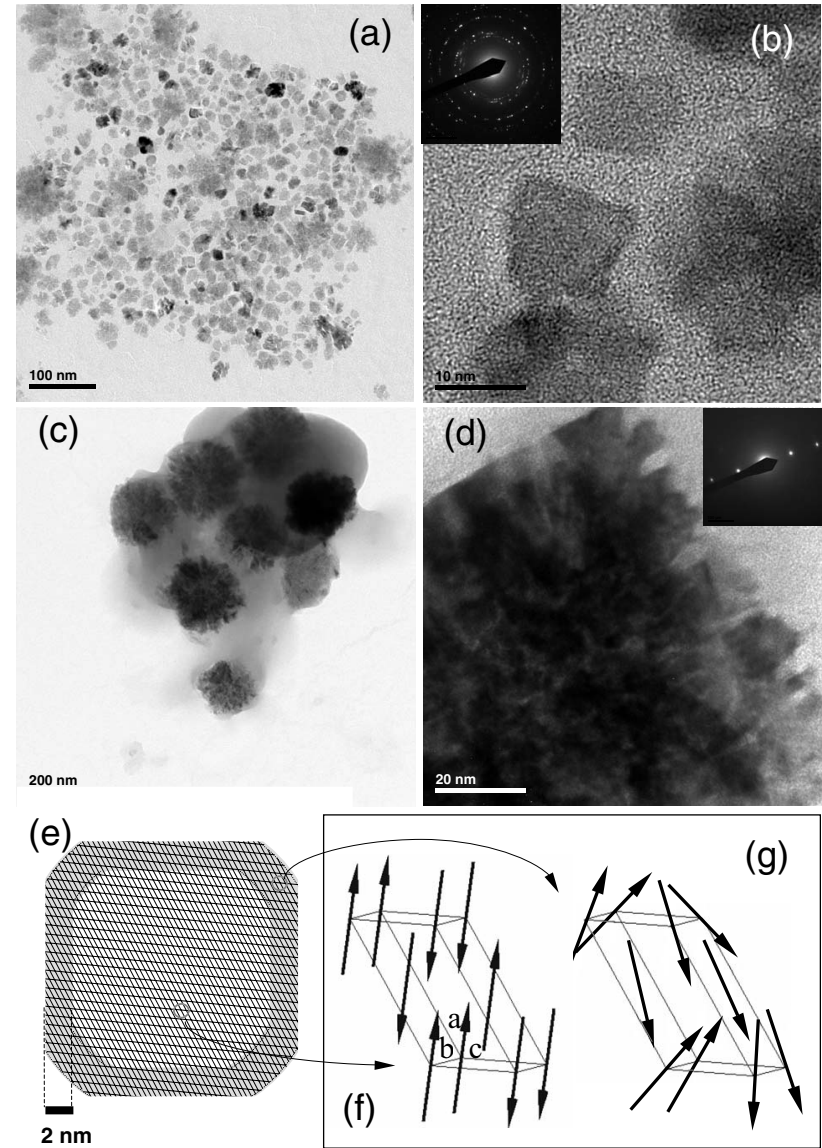

FIG. 2. (a) and (b) TEM images (scale bars correspond to 100 $\mathrm{nm}$ and $10 \mathrm{~nm}$, respectively) of the $\mathrm{CoO}$ sample with $\langle\tau\rangle_{\mathrm{XRD}}$ $=18 \mathrm{~nm}$ and inset showing an electron-diffraction image; [(c) and (d)] TEM images (scale bars correspond to $200 \mathrm{~nm}$ and $20 \mathrm{~nm}$, respectively) of the $\mathrm{CoO}$ sample with $\langle\tau\rangle_{\mathrm{XRD}}=40 \mathrm{~nm}$ and inset showing an electron-diffraction image with spots corresponding to (111) and (222) reflections; (e) schematic representation of a CoO crystallite with a magnetic disordered layer of $2 \mathrm{~nm}$; (f) magnetic structure of the nanoparticles core; and $(\mathrm{g})$ representation of the magnetic disordered surface layer.

particles size as discussed in the following section) the nuclear peaks become broader due to a monoclinic distortion (space group $\mathrm{C} 2 / \mathrm{m}$ ), ${ }^{32}$ and peaks associated to $\mathrm{AF}$ magnetic ordering appear. At $5 \mathrm{~K}$, the PND pattern can be well fit by a monoclinic nuclear cell [Fig. 1(b)] with parameters given in Table I and by a propagation vector $\mathbf{k}=[1 / 2,1 / 2,1 / 2]$ associated to a cubic cell, in accordance with Ref. 32. With respect to the monoclinic cell, $\mathbf{k}$ splits into the four vectors $\mathbf{k}_{1}=[0,1,1 / 2], \mathbf{k}_{2,3}=[1 / 2, \pm 1 / 2,0],{ }^{32}$ and $\mathbf{k}_{4}=[0,0,1 / 2]$. Best fits are obtained with $\mathbf{k}_{1}$ and $\mathbf{k}_{4}$, with the moments aligned in the $a c$ plane and making and angle $\varphi$ with the $a$ direction in the $45^{\circ}-58^{\circ}$ range and of about $18^{\circ}$, for $\mathbf{k}_{1}$ and $\mathbf{k}_{4}$, respectively. The magnetic structure corresponding to $\mathbf{k}_{1}$ is represented in Fig. 2(f). The solution with $\mathbf{k}_{1}$ is similar to that of Ref. 32, where the moments were found aligned in the ac plane with $\varphi=55^{\circ}$. The values of $\varphi$ for both $\mathbf{k}_{1}$ and $\mathbf{k}_{4}$ correspond to an angle $\phi$ between the moments and the plane defined by the ferromagnetically aligned moments [the (111) plane in the cubic cell] of about $12^{\circ}$ and $18^{\circ}$, which are 


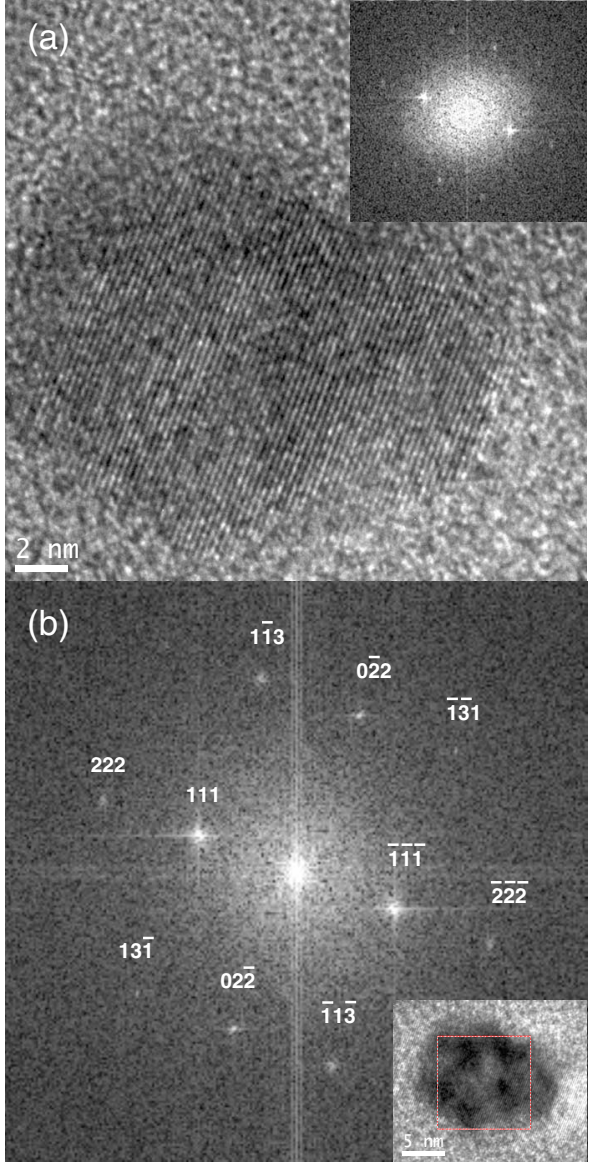

FIG. 3. (Color online) (a) HRTEM image of the CoO sample with $\langle\tau\rangle_{\mathrm{XRD}}=18 \mathrm{~nm}$ and inset showing the Fourier transform of the image; and (b) Fourier transform of the rectangular region of the image show inset.

higher than the $8^{\circ}$ found in Ref. 33 and the $1^{\circ}$ and $4^{\circ}$ recently found in Ref. 34. In fact, the magnetic structure of $\mathrm{CoO}$ is still a matter of debate, particularly in what concerns to the existence of single or multi-k structures ${ }^{35}$ and the moments direction. The present results show that the magnetic structure of the $\mathrm{CoO}$ nanoparticles is compatible to that found for bulk powder and single-crystal $\mathrm{CoO}$.

The magnetic moment per Co ion at $5 \mathrm{~K}$ for $\mathbf{k}_{1}$ in samples with $\langle\tau\rangle_{\mathrm{XRD}}=40$ and $68 \mathrm{~nm}$ is 3.41(7) and 3.40(4) $\mu_{B}$, re- spectively (Table I); similar values are obtained for $\mathbf{k}_{4}$. These values are close to those reported in Ref. $36\left(3.4 \pm 0.1 \mu_{B}\right)$ and slightly smaller than that reported in Ref. 32 $\left[3.98(6) \mu_{B}\right]$ and Ref. $37\left(3.8 \mu_{B}\right)$. All these values are higher than the spin-only moment of $3 \mu_{B}$, indicating an important orbital contribution.

As temperature increases, the magnetic (sublattice) moment $M_{\text {sub }}$ of samples $\langle\tau\rangle_{\mathrm{XRD}}=40$ and $68 \mathrm{~nm}$ decreases, similarly to that reported for bulk. ${ }^{36}$ At lower temperatures $\left(T / T_{N}<0.5\right), M_{\text {sub }}(T)$ in the bulk was described by spin waves while close to $T_{N}$ it follows a Brillouin law. At intermediate temperatures non of these models apply [see Fig. 7(b) for comparison between $M_{\text {sub }}(T)$ and Brillouin law in sample with $\langle\tau\rangle_{\mathrm{XRD}}=68 \mathrm{~nm}$ ].

In both samples, the mean size of the structurally ordered region obtained from the XRD patterns is higher than that obtained from the PND data (Table I). This might be due to uncertain associated to the determination of the instrument resolution function. At the same time, the nuclear (structural) coherence size of both samples is higher than the magnetic coherence size. In the sample with smaller size, where the contribution to the PND peaks broadening due to the sample is more relevant and thus the accuracy in the sizes determination is better, the difference between the structural and magnetic sizes is consistent in the two instruments used (5.4 $\mathrm{nm}$ and $4.4 \mathrm{~nm}$ in D20 and D1A, respectively). Considering the value obtained in the instrument with better resolution in the low angle region $(\mathrm{D} 1 \mathrm{~A})$, the $\mathrm{CoO}$ nanoparticles of sample $\langle\tau\rangle_{\mathrm{XRD}}=40 \mathrm{~nm}$ can be described by a core-shell model, where the core is structural and magnetically ordered and the shell is structurally ordered and magnetically disordered, with a thickness of about $2 \mathrm{~nm}$, corresponding to about seven nuclear cells in the $c$ direction, as depicted in Fig. 2(e).

\section{B. Size dependence of $T_{N}$}

At temperatures in the $240-290 \mathrm{~K}$ range, the magnetic susceptibility has a maximum. In the context of magnetic nanoparticles, this could correspond to a $T_{N}$ or to a blocking temperature $T_{B}$. The former is associated to the intraparticle $\mathrm{AF}$ ordering. The latter is associated to the temperature below which the magnetic moment of the nanoparticles is not

TABLE I. Nuclear and magnetic parameters (propagation vector $\mathbf{k}_{1}=[0,1,1 / 2]$ ) obtained from Rietveld refinement of neutron-diffraction data. Numbers in parenthesis denote the standard deviation expressed in units of the least significant digit. The standard deviation of the size was estimated based on the standard deviation of the fitted parameters and disregarding the uncertain associated to the determination of the parameters associated to the instrument contribution.

\begin{tabular}{|c|c|c|c|c|c|c|c|c|c|c|c|c|}
\hline \multirow[b]{2}{*}{$\begin{array}{c}\langle\tau\rangle_{\mathrm{XRD}} \\
(\mathrm{nm})\end{array}$} & \multirow[b]{2}{*}{ Instrument } & \multicolumn{7}{|c|}{ Nuclear } & \multicolumn{4}{|c|}{ Magnetic } \\
\hline & & $\begin{array}{c}T \\
(\mathrm{~K})\end{array}$ & $\begin{array}{c}a \\
(\AA)\end{array}$ & $\begin{array}{c}b \\
(\AA)\end{array}$ & $\begin{array}{c}c \\
(\AA)\end{array}$ & $\begin{array}{c}\beta \\
(\operatorname{deg})\end{array}$ & $\begin{array}{l}\text { Size } \\
(\mathrm{nm})\end{array}$ & Bragg-R & $\begin{array}{c}\mu \\
\left(\mu_{B}\right)\end{array}$ & $\begin{array}{c}\varphi \\
(\operatorname{deg})\end{array}$ & $\begin{array}{l}\text { Size } \\
(\mathrm{nm})\end{array}$ & Mag-R \\
\hline \multirow[t]{3}{*}{40} & D20 & 2.1 & $5.178(1)$ & $3.0066(5)$ & $3.0051(5)$ & $125.49(2)$ & $30.8(2)$ & 4.84 & $3.41(7)$ & $50(1)$ & $25.4(3)$ & 12.4 \\
\hline & D1A & 5.4 & $5.1895(6)$ & $3.0106(5)$ & $3.0116(5)$ & $125.464(9)$ & $24.1(2)$ & 4.25 & $3.44(7)$ & $59(1)$ & $19.7(2)$ & 5.83 \\
\hline & D20 & 295 & $4.2476(6)$ & & & & & 6.86 & & & & \\
\hline \multirow[t]{2}{*}{68} & D20 & 5.4 & $5.1828(6)$ & $3.0123(3)$ & $3.0110(3)$ & $125.512(8)$ & $57(1)$ & 9.43 & $3.40(4)$ & $44.9(7)$ & $40(1)$ & 16.9 \\
\hline & D20 & 305 & $4.2558(1)$ & & & & & 3.38 & & & & \\
\hline
\end{tabular}




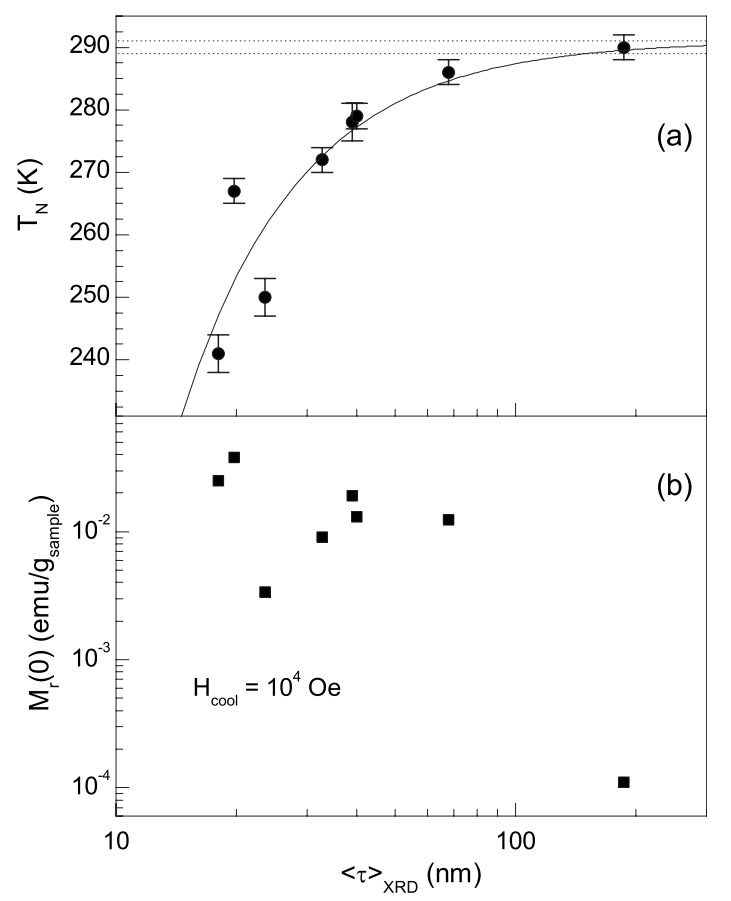

FIG. 4. (a) Dependence of the Néel temperature $T_{N}$ (determined from magnetic-susceptibility data) with the structural mean size $\langle\tau\rangle_{\mathrm{XRD}}$ (determined from Rietveld refinement to XRD data). Horizontal dotted lines delimitate the range of values for $T_{N}$ in bulk samples. Continuous line represents the fit to Eq. (1). (b) Dependence of the remanent magnetization at zero temperature $M_{r}(0)$ (obtained after cooling the samples with a field of $1 \times 10^{4} \mathrm{Oe}$ ) with $\langle\tau\rangle_{\mathrm{XRD}}$ in a log-log plot.

able to cross the anisotropy energy barrier within the experimental characteristic time, being a dynamical phenomena always associated to a frequency-dependent ac susceptibility and to the existence of an out-of-phase component. ${ }^{38}$ Since the PND measurements (which are directly probing the phase transition via the ordering parameter) performed in samples with $\langle\tau\rangle_{\mathrm{XRD}}=40$ and $68 \mathrm{~nm}$ yield $T_{N}$ values at the temperatures where the susceptibility peaks occur and since no frequency dependence or out-of-phase susceptibility is found in the 240-320 K range, the peaks of the magnetic susceptibility are associated with $T_{N}$. In fact, it is expected that within the characteristic sizes here studied $(>18 \mathrm{~nm}) T_{N}$ estimated from susceptibility peaks approaches the true thermodynamic values, in accordance to that shown for $\mathrm{CoO}$ films, where for thicknesses higher than $\sim 4 \mathrm{~nm} T_{N}$ estimated from susceptibility peaks approaches the true values measured by heat capacity. ${ }^{39}$

As the size of the nanoparticles decreases, the transition temperature $T_{N}$ (estimated from the maximum of the zfc susceptibility curve) decreases [Fig. 4(a)]. This decrease can be described in first approach by the finite-size scaling dependence

$$
\frac{T_{N}(\infty)-T_{N}(D)}{T_{N}(\infty)}=\left(\frac{\varepsilon_{0}}{\langle\tau\rangle_{\mathrm{XRD}}}\right),
$$

where $\varepsilon_{0}$ is the correlation length at $T=0$ and $\lambda_{d}$ is the shift exponent for the finite-size scaling. ${ }^{40}$ By fixing $T_{N}(\infty)$ within

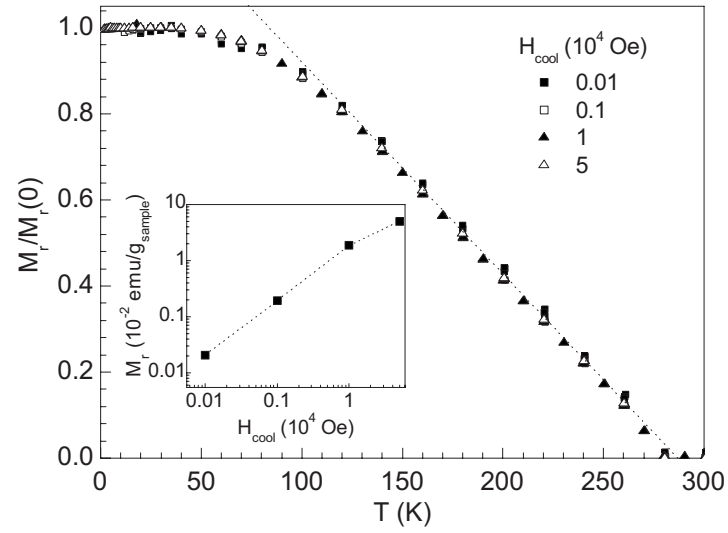

FIG. 5. Temperature dependence of the reduced remanent magnetization $M_{r} / M_{r}(0)$ of sample with $\langle\tau\rangle_{\mathrm{XRD}}=39 \mathrm{~nm}$ obtained for different cooling fields $H_{\text {cool }}$. Inset shows the dependence of $M_{r}$ at $2 \mathrm{~K}$ with $H_{\text {cool }}$.

the limits found in literature, ${ }^{36,37}$ the best fit is obtained with $\varepsilon_{0}=5 \mathrm{~nm}$ and $\lambda_{d}=1.5 . \varepsilon_{0}$ is larger than the value found in CoO films $(1.8 \mathrm{~nm})$ while the value of $\lambda_{d}$ is similar $\left(\lambda_{d}\right.$ $=1.55$ ), being between the value corresponding to those of Ising $\left(\lambda_{d}=1.56\right)$ and three-dimensional Heisenberg systems $\left(\lambda_{d}=1.42\right){ }^{41}$

These $T_{N}\left(\langle\tau\rangle_{\mathrm{XRD}}\right)$ results demonstrate that in the CoO NP, finite-size effects are relevant and contribute to the reduction in $T_{N}$, despite their aggregation state. We notice that $T_{N}$ may also be affected by the magnetically disordered layer identified by PND, although the present results are inconclusive on this respect. In the $\mathrm{CoO}$ sample from Sigma-Aldrich $T_{N}$ is within the values found in bulk samples, ${ }^{36,37}$ and therefore finite-size effects are not relevant. In the view of finite-size effects, this sample can be used as a reference for $\mathrm{CoO}$ bulk magnetic behavior.

\section{Remanent magnetization}

The remanent magnetization induced by cooling in the presence of a magnetic field, determined as described in Sec. II, saturates at low temperature and decreases almost linearly with the temperature up to temperatures near $T_{N}$ (Fig. 5). As the cooling field $H_{\text {cool }}$ increases, $M_{r}$ at $2 \mathrm{~K}$ also increases, following a power law with an exponent close to 1 , in first approach and within the studied field range (Fig. 5, inset). This contrasts with the $H^{2}$ dependence of $M_{r}$ with origin in domain walls of bulk AF materials. ${ }^{1}$ On the other hand, $M_{r}$ is still not saturated at fields much higher than those typical of AF materials with diluted impurities. ${ }^{2}$

The reduced remanent magnetization of the $\mathrm{CoO}$ NP $M_{r} / M_{r}(0)$ (with $M_{r}(0)$ taken as the value at $2 \mathrm{~K}$ ) scales with $H_{\text {cool }}$, as represented in Fig. 5 for sample with $\langle\tau\rangle_{\mathrm{XRD}}$ $=39 \mathrm{~nm}$, showing that as $H_{\text {cool }}$ increases a larger net moment is being kept in the field direction while having similar origin/mechanism.

The dependence of $M_{r}(0)$ with size is scattered [Fig. 4(b)]. However, it is clear that in the nanoparticles $M_{r}(0)$ is enhanced compared to bulk and in the nanoparticles where $\langle\tau\rangle_{\mathrm{XRD}}$ is about one order of magnitude lower than that of 

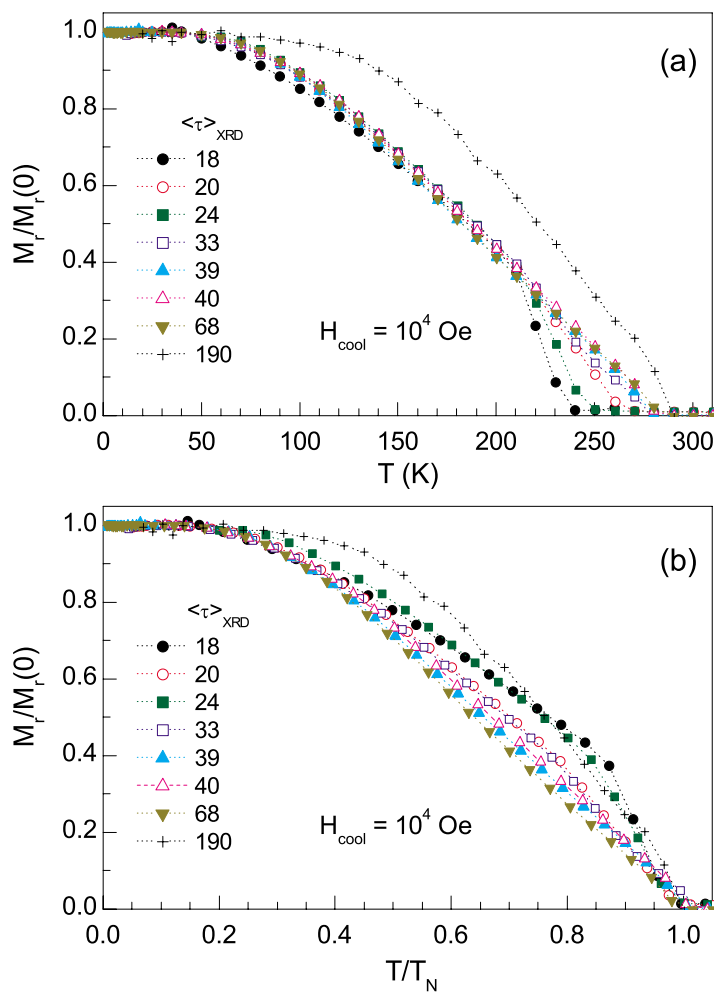

FIG. 6. (Color online) (a) Temperature dependence of the reduced remanent magnetization $M_{r} / M_{r}(0)$ of all studied samples obtained after cooling under $H_{\text {cool }}=1 \times 10^{4}$ Oe. (b) $M_{r} / M_{r}(0)$ of all studied samples obtained after cooling under $H_{\text {cool }}=1 \times 10^{4}$ Oe as a function of the reduced temperature $T / T_{N}$.

bulk, $M_{r}(0)$ is about two orders of magnitude higher.

Comparing $M_{r} / M_{r}(0)$ for the different studied sizes [Fig. $6(\mathrm{a})]$, it is interesting to note that it scales for the samples containing nanoparticles in the low and medium temperature range (in the saturation and linear region), separating only near $T_{N}$ since the samples have different $T_{N}$ values. This scaling is far better than the scaling in a $M_{r} / M_{r}(0)$ vs $T / T_{N}$ scale [Fig. 6(b)]. In fact, in all samples the linear region of $M_{r} / M_{r}(0)$ extrapolates to zero remanent magnetization for temperatures near to the bulk $T_{N}$. At low/medium temperatures, the remanent moments behave as there was no reduction in $T_{N}$ due to size effects; in other words, finite-size effects are relevant only near $T_{N}$.

$M_{r} / M_{r}(0)$ of the sample taken as bulk does not scale in $T$ nor $T / T_{N}$, showing that in this sample $M_{r}$ has a different origin, probably domain walls, as usually found in bulk AF. ${ }^{1}$ Also, the temperature dependence of $M_{r}$ is different from that arising from interfacial uncompensated moments in $\mathrm{CoO}$ films, where a plateau up to $200 \mathrm{~K}$ was found. ${ }^{4}$

In order to have a better insight on the origin of the moments associated to the $M_{r}$ induced by $H_{c o o l}$ we have performed PND experiments as a function of temperature at zero field, after zfc and after field cooling across $T_{N}$, in samples $\langle\tau\rangle_{\mathrm{XRD}}=40$ and $68 \mathrm{~nm}$. It is clear that PND patterns taken after zfc and fc are equal, within the experimental precision [Fig. 7(a)]. Since the integral breadth of the magnetic peaks remains unchanged by fc, the size of the magnetically ordered core also remains unchanged, within the precision of
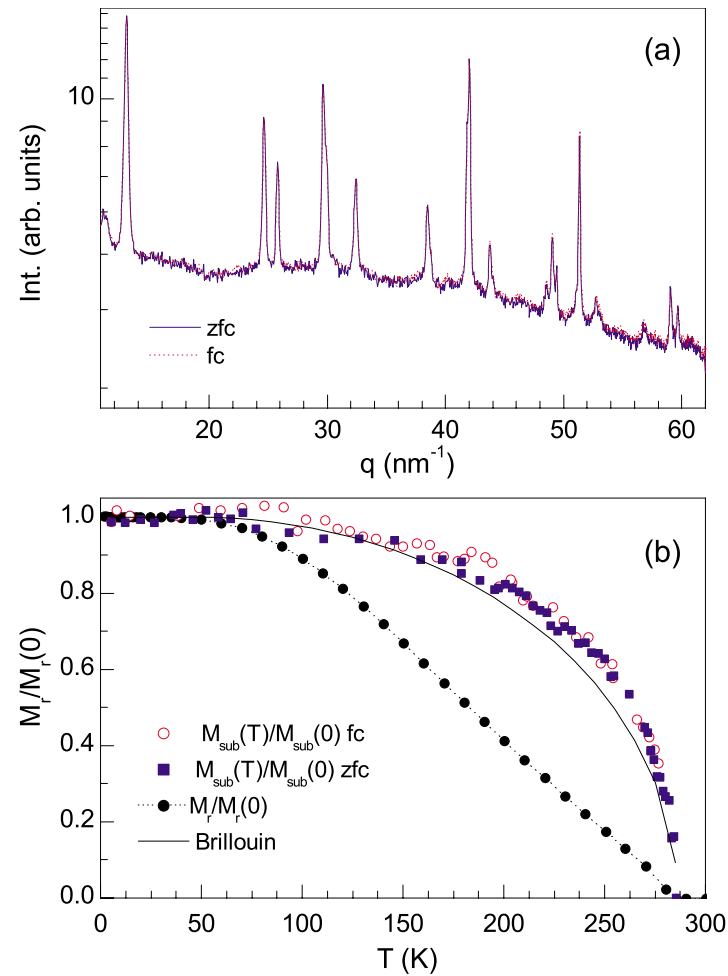

FIG. 7. (Color online) (a) PND pattern of the $\mathrm{CoO}$ nanoparticles (sample with $\langle\tau\rangle_{\mathrm{XRD}}=68 \mathrm{~nm}$ ) at $5 \mathrm{~K}$ after $\mathrm{zfc}$ and $\mathrm{fc}$ (instrument D20). (b) Temperature dependence of the reduced remanent magnetization $M_{r} / M_{r}(0)$ of sample with $\langle\tau\rangle_{\mathrm{XRD}}=68 \mathrm{~nm}$ compared to the reduced sublattice magnetization $M_{\text {sub }} / M_{\text {sub }}(0)$ obtained from Rietveld refinement to zfc and fc PND patterns. Continuous line represents the Brillouin law for $T_{N}=286 \mathrm{~K}$ and $S=3 / 2$.

a few standard deviations (see standard deviations in Table I). In this view, $M_{r}$ arises most probably from magnetic moments located at the magnetically disordered surface and not from an important change in the size of the magnetically ordered core induced by fc. The surface origin of $M_{r}$ is also supported by the linear behavior of $M_{r}(T)$ at intermediate temperatures. In fact, the linear $M_{r}(T)$ indicates that the moments responsible for the remanent magnetization are in a two-dimensional (2D) configuration as experimentally 42,43 and theoretically ${ }^{44}$ found in films and particles. ${ }^{19,45}$ Furthermore, Makhlouf et al. ${ }^{16}$ refer to molecular-field calculations showing that the linearity holds down to $T / T_{N}=0.2$, similarly to that found here [Fig. 6(b)].

From the point of view of the $M_{r}(T)$ dependence, it is expected that a $2 \mathrm{~nm}$ thick shell behaves as a 2D entity based on models and experimental results. Models show that $M(T)$ depends on the number of atomic layers $n$, on the spin value and on the ratio between the parallel magnetic anisotropy constant and the exchange constant. ${ }^{44}$ For some typical values of this ratio, it is shown that for $n>10, M(T)$ become similar to bulk. In the present case of $\mathrm{CoO}, 2 \mathrm{~nm}$ correspond to about 7 Co layers, which is within the range where a shell is expected to behave as a $2 \mathrm{D}$ entity. An experimental study in $\mathrm{La}_{0.7} \mathrm{Sr}_{0.3} \mathrm{MnO}_{3}$ films ${ }^{43}$ show that $M(T)$ probed in a $5 \mathrm{~nm}$ thick surface is still substantially different from that of bulk and conclude that at least several monolayers below a $0.5 \mathrm{~nm}$ surface boundary $M(T)$ is considerably different from bulk. 


\section{CONCLUSIONS}

In this paper we show that a remanent moment $M_{r}$ can be induced in $\mathrm{CoO}$ nanoparticles by crossing the transition temperature in the presence of a magnetic field, with $M_{r}$ being approximately proportional to the cooling field. The value of $M_{r}$ at low temperature in the nanoparticles is about two orders of magnitude higher than the value found in the sample taken as the bulk. The nanoparticles are well described by a core-shell model with the following characteristics (i) the core is magnetically similar to that of the bulk (concerning both the value and direction of the magnetic moment), (ii) the core remains unchanged by cooling across $T_{N}$ in the presence of a magnetic field compared to the zero-field cooling situation within the instrumental precision, (iii) both core and shell are structurally ordered, (iv) the shell is structurally ordered and magnetically disordered having a thickness of about $2 \mathrm{~nm}$, and (v) most probably $M_{r}$ arises from pinned uncompensated/canted moments located at the shell. Points (iii) and (iv) are supported by PND and point (iii) is also in accordance with HRTEM images which show that the nanoparticles are single domain and the crystalline planes are ordered across the nanoparticles up to near the surface. Point (v) is supported by the linear dependence of $M_{r}$ with temperature. The existence of a structurally ordered and magnetically disordered shell opens the question of the source of this magnetic disorder. This source can be structural, includ- ing preferential surface vacancies (which lead to broken exchange paths and oxidized nonmagnetic $\mathrm{Co}$ ) and surface tension. Magnetic sources include an odd number of planes and the symmetry break of the exchange paths of surface atoms. The latter gives rise to a progressively canting of the moments. ${ }^{46}$ Another magnetic source for the generation of a pinned moment was discussed for NiO.${ }^{17}$ Accordingly to numerical modeling, the reduced coordination of surface spins leads to different sublattice configurations, which causes changes in the magnetic order throughout the particle. This last cause is clearly compatible with the present experimental findings.

\section{ACKNOWLEDGMENTS}

We acknowledge the collaboration of Thomas Hansen in the experiments at D20 and ILL for beamtime allocation. We also acknowledge R. Soares for the XRD patterns and A. Ferreira for enlightening comments about XRD instrumental contribution. The Aveiro-Zaragoza collaboration has been supported by the Integrated Spanish-Portuguese Action under Grant No. PT2009-0131. The work in Zaragoza has been supported by the research Grants No. MAT2007-61621, No. MAT2009-13977-C03-01, and No. CONSOLIDER CSD2007-00010 from the Ministry of Education. N.J.O.S. acknowledges CSIC for a I3P contract and FCT for Ciencia 2008 program.
*Present address: CICECO, Universidade de Aveiro, 3810-193 Aveiro, Portugal; nunojoao@ua.pt

${ }^{1}$ P. Pollak, W. Kleemann, and D. P. Belanger, Phys. Rev. B 38, 4773 (1988).

${ }^{2}$ F. Palacio, M. Gabás, J. Campo, C. C. Becerra, A. Paduan-Filho, and V. B. Barbeta, Phys. Rev. B 56, 3196 (1997).

${ }^{3}$ Ò. Iglesias, A. Labarta, and X. Batlle, J. Nanosci. Nanotechnol. 8, 2761 (2008).

${ }^{4}$ K. Takano, R. H. Kodama, A. E. Berkowitz, W. Cao, and G. Thomas, Phys. Rev. Lett. 79, 1130 (1997).

${ }^{5}$ A. E. Berkowitz, G. F. Rodriguez, J. I. Hong, K. An, T. Hyeon, N. Agarwal, D. J. Smith, and E. E. Fullerton, Phys. Rev. B 77, 024403 (2008).

${ }^{6}$ W. H. Meiklejohn and C. P. Bean, Phys. Rev. 105, 904 (1957).

${ }^{7}$ V. Skumryev, S. Stoyanov, Y. Zhang, G. Hadjipanayis, D. Givord, and J. Nogués, Nature (London) 423, 850 (2003).

${ }^{8}$ I. V. Golosovsky, G. Salazar-Alvarez, A. López-Ortega, M. A. González, J. Sort, M. Estrader, S. Suriñach, M. D. Baró, and J. Nogués, Phys. Rev. Lett. 102, 247201 (2009).

${ }^{9}$ A. H. Morrish and K. Haneda, J. Magn. Magn. Mater. 35, 105 (1983).

${ }^{10}$ T. Okada, H. Sekizawa, F. Ambe, S. Ambe, and T. Yamadaya, J. Magn. Magn. Mater. 31-34, 903 (1983).

${ }^{11}$ F. T. Parker and A. E. Berkowitz, Phys. Rev. B 44, 7437 (1991).

${ }^{12}$ F. T. Parker, M. W. Foster, D. T. Margulies, and A. E. Berkowitz, Phys. Rev. B 47, 7885 (1993).

${ }^{13}$ A. E. Berkowitz, J. A. Lahut, and C. E. Van Buren, IEEE Trans. Magn. 16, 184 (1980).
${ }^{14}$ P. Guardia, B. Batlle-Brugal, A. G. Roca, Ò. Iglesias, M. P. Morales, C. J. Serna, A. Labarta, and X. Batlle, J. Magn. Magn. Mater. 316, e756 (2007).

${ }^{15}$ M. M. Ibrahim, J. Zhao, and M. S. Seehra, J. Mater. Res. 7, 1856 (1992).

${ }^{16}$ S. A. Makhlouf, F. T. Parker, and A. E. Berkowitz, Phys. Rev. B 55, R14717 (1997).

${ }^{17}$ R. H. Kodama and A. E. Berkowitz, Phys. Rev. B 59, 6321 (1999).

${ }^{18}$ A. Punnoose, H. Magnone, M. S. Seehra, and J. Bonevich, Phys. Rev. B 64, 174420 (2001).

${ }^{19}$ D. L. Mills, Phys. Rev. B 3, 3887 (1971).

${ }^{20}$ A. H. Morrish and K. Haneda, J. Appl. Phys. 52, 2496 (1981).

${ }^{21}$ K. Haneda and A. H. Morrish, J. Appl. Phys. 63, 4258 (1988).

${ }^{22}$ Z. X. Tang, C. M. Sorensen, K. J. Klabunde, and G. C. Hadjipanayis, Phys. Rev. Lett. 67, 3602 (1991).

${ }^{23}$ D. Lin, A. C. Nunes, C. F. Majkrzak, and A. E. Berkowitz, J. Magn. Magn. Mater. 145, 343 (1995).

${ }^{24}$ B. Martínez, X. Obradors, Ll. Balcells, A. Rouanet, and C. Monty, Phys. Rev. Lett. 80, 181 (1998).

${ }^{25}$ S. Linderoth, P. V. Hendriksen, F. Bødker, S. Wells, K. Davies, S. W. Charles, and S. Mørup, J. Appl. Phys. 75, 6583 (1994).

${ }^{26}$ A. Millan, A. Urtizberea, F. Palacio, N. J. O. Silva, V. S. Amaral, E. Snoeck, and V. Serin, J. Magn. Magn. Mater. 312, L5 (2007).

${ }^{27}$ X. Sun, Y.-W. Zhang, R. Si, and C.-H. Yan, Small 1, 1081 (2005).

${ }^{28}$ M. Ghosh, E. V. Sampathkumaran, and C. N. R. Rao, Chem. Mater. 17, 2348 (2005). 
${ }^{29}$ J. Rodríguez-Carvajal, Physica B 192, 55 (1993).

${ }^{30}$ D. Martínez-Blanco, P. Gorria, J. A. Blanco, M. J. Pérez, and J. Campo, J. Phys.: Condens. Matter 20, 335213 (2008).

${ }^{31}$ J. D. Hanawalt, H. W. Rinn, and L. K. Frevel, Ind. Eng. Chem. Anal. Ed. 10, 457 (1938).

${ }^{32}$ W. Jauch, M. Reehuis, H. J. Bleif, F. Kubanek, and P. Pattison, Phys. Rev. B 64, 052102 (2001).

${ }^{33}$ D. Herrmann-Ronzaud, P. Burlet, and J. Rossat-Mignod, J. Phys. C 11, 2123 (1978).

${ }^{34}$ E. Ressouche, N. Kernavanois, L.-P. Regnault, and J.-Y. Henry, Physica B 385-386, 394 (2006).

${ }^{35}$ K. Tomiyasu, T. Inami, and N. Ikeda, Phys. Rev. B 70, 184411 (2004).

${ }^{36}$ M. D. Rechtin and B. L. Averbach, Phys. Rev. B 6, 4294 (1972).

${ }^{37}$ W. L. Roth, Phys. Rev. 110, 1333 (1958).
${ }^{38}$ J. L. Dormann, D. Fiorani, and E. Tronc, Advances in Chemical Physics (Wiley, New York, 1997), Vol. 98.

${ }^{39}$ Y. J. Tang, D. J. Smith, B. L. Zink, F. Hellman, and A. E. Berkowitz, Phys. Rev. B 67, 054408 (2003).

${ }^{40}$ M. E. Fisher and M. N. Barber, Phys. Rev. Lett. 28, 1516 (1972).

${ }^{41}$ T. Ambrose and C. L. Chien, Phys. Rev. Lett. 76, 1743 (1996).

${ }^{42}$ G. Xiao, C. L. Chien, and M. Natan, J. Appl. Phys. 61, 4314 (1987).

${ }^{43}$ J.-H. Park, E. Vescovo, H.-J. Kim, C. Kwon, R. Ramesh, and T. Venkatesan, Phys. Rev. Lett. 81, 1953 (1998).

${ }^{44}$ A. Corciovei, Phys. Rev. 130, 2223 (1963).

${ }^{45}$ Ò. Iglesias and A. Labarta, Phys. Rev. B 63, 184416 (2001).

${ }^{46}$ L. Néel, J. Phys. Soc. Jpn. 17, Suppl. B, 676 (1962). 